\title{
Performance Evaluation of an AODV-Based Routing Protocol Implementation by Using a Novel In-Field WSN Diagnosis Tool
}

\author{
Gabriel Mujica, Rafael Zamacola, Jorge Portilla, Teresa Riesgo
}

\begin{abstract}
The Wireless Sensor Network research field has been growing and becoming more mature during the last decade since novel technologies and research lines have emerged targeting its usability in the real world under different application scenarios. One of the key topics to assure the efficiency and effectiveness of these technologies in final applications is the communication and routing strategies within the WSN. In this context, it is essential to evaluate and validate the implementation of routing algorithms and network connectivity in real deployments, as a support of theoretical simulation models that cannot predict certain constraints and limitations in the behavior of the system. In this way, a real implementation of a flexible AODV-based routing protocol using a modular HW-SW node platform is proposed in this work, in addition to its practical assessment under real conditions by using a novel in-situ WSN evaluation tool. This tool has been created to support users during the deployment analysis and diagnosis in real environments, beyond the typical study of routing performance through WSN simulators.
\end{abstract}

Keywords - wireless sensor networks, routing protocols, network diagnosis, HW-SW co-design.

\section{INTRODUCTION \& RELATED WORK}

During the last decade the Wireless Sensor Networks (WSNs) have emerged as a key and challenging topic for the research community due to the inherent integration of a wide range of different hardware and software technologies, protocols and new algorithms in order to maximize their performance and feasibility in final applications. One of most relevant aspects to be considered to assure the efficiency and effectiveness of these technologies in real scenarios is not only the quality of the service of the whole implemented system, but also the robustness and reliability of the network performance, which strongly depends on the communication capabilities as well as topologies and routing strategies within the WSN. In this context, routing problems in WSNs have been studied from different perspectives, depending on the target application, topologies needed for specific scenarios and environments, network mobility and scalability, and data aggregation [1].

On one hand, from the point of view of the network structure to be adopted, three major groups of routing algorithms can be distinguished. The first one is the flat-based routing protocols [2], in which nodes have the same role in order to collaborate in disseminating the sensor information (different route alternatives), mainly targeting unstable environments where the quality of the links are prone to be affected. The second group is the hierarchical-based routing protocols, in which two main types of roles are defined, based on cluster nodes that retransmit the measurements from sensor (end) nodes [3]. These routing protocols are mainly intended to be used in stable environments where the probability of communication failures is lower. The third group is focused on location-based protocols, in which nodes find the routing alternatives by knowing their localization (using signalstrength-based algorithms or adding GPS-based hardware) with respect to the surrounding neighbor nodes [4].

On the other hand, considering how routes are created, routing algorithms can also be classified in proactive, which mechanism periodically or continuously attempts to determine the routes even if they are not used, so that the corresponding path is already available whenever a node needs to send a packet to a destination point [5]. Although this schema reduces the time delay when a packet has to be transmitted, it has poor performance in unstable environments where the routes are prone to be changed, in which the frequency of the connectivity reconfiguration is much higher. In order to cope with these limitations, a second classification is included, the reactive protocols, in which the route determination is performed on demand [5].

Most of the aforementioned mechanisms and the state-ofthe-art routing protocols have been evaluated by using simulation tools and modeling process, which gives to some extent an idea of what the functionality of the mechanism will be. However, fewer works focus on implementing and integrating routing protocols in real hardware platforms in order to analyze the performance and the efficiency of those implementations under real conditions, as well as deal with the resource limitations of sensor nodes. In this context, it is essential to evaluate and validate the implementation of routing algorithms and network connectivity in real deployments, as a support of theoretical simulation models that cannot predict certain constraints and limitations in the behavior of the system. These are the main reasons why a real implementation of a flexible AODV-based (Ad hoc OnDemand Distance Vector) routing protocol using a modular HW-SW platform has been deeply studied and is proposed in this work, as a support of the Cookie nodes [6] and their integration with an IEEE 802.15.4 based communication layer. AODV is a flat-based routing protocol, which provides Cookies with a greater flexibility as well as allows the use of any required topology by the target application. It is also within the reactive protocol classification, which is more suitable for reconfigurable and Ad-hoc network topologies. The proposed implementation, called CB-AODV (CookieBased AODV), aims to help developers to analyze different aspects, constrains and requirements in the use of reactive routing protocols in real WSN application scenarios.

Such approach brings another important issue for the success of the implementation of routing algorithms. Nowadays there is a lack of well-defined practical tools to analyze and evaluate the implementation of routing protocols and communication topologies in WSNs within their real operational environments and final application scenarios. Furthermore, the behavior of routing protocols running in actual hardware platforms might be far from the expected simulation results, due to limitations concerning the core and memory architecture on which routing algorithms are to be implemented, as well as computational and processing limitations that are not taken 
into account during the modeling process. In this work, a practical assessment of routing protocols (and specially for the CB-AODV implementation) under real conditions is also proposed as another major contribution, by means of creating a novel in-situ WSN evaluation tool (I-PEDT, In-situ Performance Evaluation and Diagnosis Tool). This tool is proposed to support users during the in-field deployment analysis and diagnosis in order to correlate theoretical results with the operation of the network in real environments, beyond the typical study of routing performance through WSN simulators. There are works focused on coping with the evaluation of routing protocols from a simulation perspective, such as the framework presented in [7]. However, fewer proposals try to address practical assessment of network connectivity and in-field analysis of routing mechanisms, such as the work presented in [8], or general approaches for evaluating node functionalities [9].

The rest of the paper is organized as follows, starting with the CB-AODV implementation and its integration with the HW-SW platform in section 2. In section 3 a discussion regarding the proposed in-situ Evaluation Tool is presented, whereas in section 4 experimental results are analyzed in detail. Finally, conclusions and contributions are provided.

\section{CB-AODV IMPLEMENTATION}

In order to deeply analyze and study network connectivity issues in WSNs, a new communication layer has been designed and implemented based on the IEEE 802.15.4 standard [10] (which is the most common communication standard used in WSNs), taking advantages of the modularity and the ease of integration of the Cookie platform [6], as shown in figure 1. The selected module is the well-known CC2420 from Texas Instruments [11], which has been widely used in different node platforms.

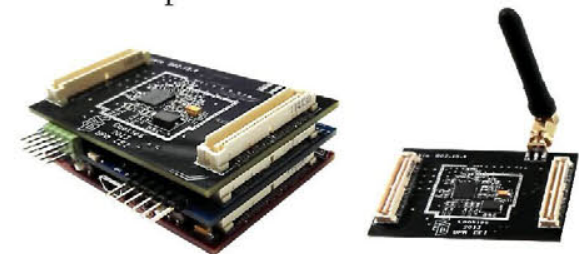

Figure 1. IEEE 802.15.4-based Cookie communication layer.

On the other hand, based on the inherent flexibility of the Cookie nodes to be adapted to different WSN application contexts, and also according to the state-of-the-art analysis of the previous section, the AODV-based routing protocol [12] has been selected as the most suitable algorithm to be integrated into the HW-SW platform, due to its adaptability to different network environments. Taking advantages of the benefits of this routing protocol, a modified version is proposed to be adapted and implemented in a real HW-SW node platform (named CB-AODV), as described in the following subsections.

\subsection{Basic operation of the protocol}

The AODV protocol uses 4 different types of control messages to create and maintain the routes of the network. These messages are named RREQ (route request message), RREP (route reply message), RERR (route error message) and HELLO. Moreover, every node of the network keeps a routing table with different fields that include information for the maintenance of the routes. When a node decides to send an application packet to another remote node of the network, the first step is to check if it has the route to the destination point in its own routing table. If so, the message is then sent to the next node of the route. This is repeated along the whole path until the packet reaches the final destination. In case that the node does not have information of the route, it will store the packet and start the route request process by broadcasting a RREQ message. The devices that receive this message will rebroadcast it until one of the nodes has information of the route, or the destination itself is the one that receives such request message. In both cases the node will send a RREP to the previous node until it reaches the source node that requested the route, and then the application packet previously stored is finally sent through the discovered path. In this way, with the propagation of the RREQ, the nodes update their corresponding routing table with the information of the source node, thus creating what is called the inverse route. In addition to this, nodes also create a route table entry with the information of the node from which they received the RREQ, hence creating routes to their neighbor nodes. With the propagation of the RREP, a direct route is created, which implies that the different nodes where the RREP hops create a route to the destination point. The computed routes are only valid during a limited period of time, and once they are not used within this timeout they are marked as invalid, and therefore they are no longer available for use. In these cases, or due to a link failure in the propagation of a data packet (no acknowledge received in any of the attempts to transmit a packet to the next hop), the node that detects the failure sends a RERR to all of its precursors nodes, i.e., those nodes that use the former node as their next hop. The precursors will then repeat the same process until the nodes that used the fail route delete it from their routing table.

\section{2. $C B-A O D V-H W-S W$ adaptation}

The AODV routing protocol was initially implemented to be used in MANET networks (mobile ad-hoc networks). Despite all the similarities with respect to the WSNs, the wireless nodes have less processing capabilities and the battery autonomy has to be taken into account in order to enhance the overall lifetime of the deployment. Because of that, it is necessary to adapt the protocol to the WSN characteristics and especially to the architecture of the Cookie HW-SW platform and its usage in real application scenarios. Therefore, the proposed adaptation is focused on adding new functionalities to the original protocol and not including some others aiming to improve the overall performance of the algorithm for the purpose of the Cookie node connectivity and reducing the resource consumption within the system architecture. The main adaptations are classified as follows.

Reduction of the control message headers and the routing tables. This is one of the most suitable optimizations because many of the fields of the message frames are not used and some of them can be reduced for most of the applications.

Elimination of the time to live of the routes and suppression of HELLO messages. With this optimization it is possible to reduce overhead of the network (there are not messages to be sent periodically for route maintenance). Moreover, it is not needed to expend additional resources to check if the routes have been used for a period of time, thus reducing the implementation size.

Expanding Ring Search algorithm removed. This algorithm is based on searching the routes by using RREQ messages with a low TTL (time to live, i.e., maximum number of hops that the message can do during the discovery process). If no RREP is received, then the RREQ is rebroadcasted and the TTL is increased until the route is discovered or the TTL has reached its maximum value. This algorithm is used to avoid flooding the network at the expense of increasing the latency. 
In addition to the aforementioned modifications, additional features have also been included in CB-AODV in order to increase its performance without penalizing the limited memory resources of the architecture, as described below:

Implementation of different types of metrics: The discovery metric is a fundamental parameter used to compare the different route alternatives that can be obtained during a route discovery process. The metric applied in the AODV protocol is the number of hops (i.e., as less number of hops as possible to determine what route is the best), which has poor performance in unstable and "crowded" WSN application environments. Therefore, it is fundamental to consider other types of metrics that can obtain the best routes in terms of the quality of the interconnections among the wireless nodes. For this purpose, metrics that use a parameter called LDR (Link Delivery Ratio) for measuring the percentage of messages that will be successfully sent between 2 nodes are considered. The LDR can be calculated directly or indirectly with the LQI parameter provided by the IEEE 802.15.4 standard. In the Cookies implementation, a correlation between the LQI and the LDR for the CC2420 radio transceiver has been used and based on the analysis proposed in [13], the metrics that have been included in order to evaluate their performance in the Cookie platform are the followings:

- PATH-DR (Path Delivery Ratio) metric: this metric calculates the percentage of messages that theoretically will be successfully sent in a route. It is based on the LDR parameter.

- ETX (Expected Transmission Count) metric: tries to minimize the number of hops by taking into account the possibility of link failures due to the quality of the LDR parameter.

- Number of hops: as explained before, it is the original metric used in the protocol, which consumes less memory resources due to its ease of calculation and implementation.

New type of message: In addition to the included features, in this work a novel type of message has been implemented for the CB-AODV protocol, called DATA_ERR. Whenever an intermediate node discovers a link failure when transmitting a data packet, after sending the RERR it will also send the originate message to the source node indicating that it is a DATA_ERR message. When the originator of the message receives the DATA_ERR, it will store the data packet and then try to discover an alternative route to send the message, so that an information integrity process is performed in order to avoid losing critical data in unstable WSN application environments.

\subsection{Non-included functions}

There are also other types of adaptations to reduce the need of memory and processing capabilities that are not included in CB-AODV, because they also reduce the performance of the protocol and were not necessary within the Cookie platform context, where a trade-off between performance efficiency and resource constraints is adopted to assure its flexibility in different application scenarios, as described below:

Suppression of the Local Repair mechanism. The Local Repair is a functionality in which an intermediate node discovers a link failure when sending a message and, instead of sending a RERR message, it will try to find an alternate route. If the local repair mechanism is deleted it is possible to reduce the consumed memory because an output queue is no longer needed, however it decreases the protocol performance.

Only the final destination is capable of generating RREP messages. This has the advantage that no loops (circular paths in which the message continuously hops never reaching the destination) are made because the intermediate nodes cannot generate RREPs. The sequence number is a field in the routing table and in the control messages created to avoid the generation of loops, so if no loops can appear, then sequence numbers are no longer needed. Moreover, it is possible to eliminate the use of RERR messages to decrease the complexity of the protocol.

Suppression of the metrics. If no metric is used, the node only generates a RREP for the first received RREQ and not for the rest that it might receive during the discovery process (even if they are better routes). This option allows reducing memory in the routing tables and the size of the control message frames, but decreases the effectiveness and robustness of the protocol.

Precursor list. As explained before, the RERR messages are sent to the precursors of the route that has failed, so it is necessary to have a list of possible precursors for every routing table entry, which increases the memory usage. However, if precursors are not implemented, the propagation of the RERR messages is similar to the propagation of RREQ messages, which floods the network and they might cause the suppression of routes that did not need to be eliminated.

\subsection{Comparison of AODV implementations and proposals}

In table 1 (extracted from [14]) a comparison among different AODV implementations and proposals is shown in detail, in which the proposed CB-AODV has been added.

CB-AODV has very similar features compared to the NSTAODV implementation, being the ones with more characteristics from the original AODV as defined in the RFC and, therefore, the ones that will theoretically have better performance. The main difference is that the former one incorporates the precursor list, allowing the protocol to have better efficiency in the propagation of the RERR messages, as well as the inclusion of the DATA_ERR message, thus increasing the performance of the implementation. Another important optimization is also proposed in terms of memory usage. For those applications in which the size of the network is less than 255 nodes, the control message frames can be reduced by means of using a 1-byte-based address field. In this case, the memory space of the routing tables can be also reduced and fixed. The latest option aims to increase the performance of the protocol since the latency due to routing table entry searching is reduced (memory access) by assigning a predefined position in the table entries for every node.

\subsection{Loop problems}

Another key aspect that has been taken into account for the success of the CB-AODV implementation is the possible generation of loops. As explained before, the AODV protocol includes the sequence numbers to avoid this problem. However, the RFC 3561 has some ambiguities and some of the interpretations can yield loops, as detailed in [15]. Based

Table 1. Comparison of AODV implementations and proposals.

\begin{tabular}{|c|c|c|c|c|c|c|c|}
\hline & $\begin{array}{l}\text { Connectivaty } \\
\text { maintenance } \\
\text { mechanism }\end{array}$ & $\begin{array}{l}\text { RERR } \\
\text { message }\end{array}$ & $\begin{array}{l}\text { Precursor } \\
\text { list }\end{array}$ & $\begin{array}{l}\text { Local } \\
\text { repair }\end{array}$ & $\begin{array}{c}\text { Only } \\
\text { destination } \\
\text { generates } \\
\text { RREP } \\
\end{array}$ & $\begin{array}{l}\text { Routhing } \\
\text { metric }\end{array}$ & $\begin{array}{l}\text { Specthication } \\
\text { ond/or } \\
\text { implementation } \\
\text { status } \\
\end{array}$ \\
\hline $\begin{array}{l}\overline{A O D V R F C} \\
3561\end{array}$ & $\begin{array}{l}\text { Hellomessage, } \\
\text { uN, etc. }\end{array}$ & Yes & yes & $\begin{array}{l}\text { Yes } \\
\text { (may) }\end{array}$ & No & Hop count & $\begin{array}{c}\mathrm{RFC} \\
\text { experimental }\end{array}$ \\
\hline AODV jr & $\begin{array}{c}\text { Connect } \\
\text { messages } \\
\text { (unidirectional } \\
\text { traffic) }\end{array}$ & No & No & No & yes & Fastest RREP & $\begin{array}{l}\text { Implemented } \\
\text { forthe ns-2 } \\
\text { simulator }\end{array}$ \\
\hline AODV bis & $\begin{array}{l}\text { Hellomessage, } \\
\text { UN, etc. }\end{array}$ & $\begin{array}{c}\text { Yes } \\
\text { (should) }\end{array}$ & No & No & No & $\begin{array}{l}\text { Fastest RREP } \\
\text { (hop count } \\
\text { is } \\
\text { optional) }\end{array}$ & $\begin{array}{l}\text { Expired Internet } \\
\text { Draft }\end{array}$ \\
\hline $\begin{array}{l}\text { LOWPAN- } \\
\text { AODV }\end{array}$ & UN & No & No & No & yes & Hop count & $\begin{array}{l}\text { Expired internet } \\
\text { Draft }\end{array}$ \\
\hline LOAD & แN & $\begin{array}{l}\text { Yes } \\
\text { (may) }\end{array}$ & No & $\begin{array}{c}\text { Yes } \\
\text { (may) }\end{array}$ & $\begin{array}{l}\text { Yes } \\
\text { (should) }\end{array}$ & Lar-based & InternetDraft \\
\hline TinYAODV & $\begin{array}{l}\text { ULN (disabled } \\
\text { by default) }\end{array}$ & yes & No & No & yes & Hop count & $\begin{array}{l}\text { Implemented } \\
\text { for Tinyos }\end{array}$ \\
\hline NST-AODV & uN & Yes & No & yes & No & $\begin{array}{l}\text { Multiple } \\
\text { metrics }\end{array}$ & $\begin{array}{l}\text { Implemented } \\
\text { for Tinyos }\end{array}$ \\
\hline CB-AODV & LLN & yes & yes & yes & No & $\begin{array}{l}\text { Multiple } \\
\text { metrics }\end{array}$ & $\begin{array}{l}\text { Implemented } \\
\text { for the Cookie } \\
\text { HW-sW } \\
\text { platform }\end{array}$ \\
\hline
\end{tabular}


on this, there are 4 different interpretations that can cause the generation of loops. In the proposed CB-AODV implementation the selected options are $1 \mathrm{~b}, 2 \mathrm{c}, 3 \mathrm{~d}$ y $4 \mathrm{c}$ [15]. It is important to highlight that the option 4 does not produce loops because in option 3 self-entries are not allowed.

\section{I-PEDT: IN-SITU PERFORMANCE EVALUATION TOOL}

The performance of routing protocols under realistic conditions is essential to assure the effectiveness and the reliability of the final application where nodes are being used. However, as previously explained, there is a lack of welldefined practical tools to analyze and evaluate the implementation of routing protocols and communication topologies in WSNs under real conditions and final deployments, beyond the simulation/emulation of the modeled algorithms and metrics. Realistic environments include unpredictable situations that cannot be covered in simulation models. Moreover, the behavior of routing protocols running in actual hardware platforms might be far from the expected simulation results, due to limitations concerning the core and memory architecture where routing algorithms are to be implemented as well as computational and processing limitations that are not taken into account during the modeling process. Therefore, it is crucial to establish an in-field based support mechanism to test and assess a WSN in terms of connectivity and routing capabilities in real deployment scenarios, thus allowing developers to analyze and compare functionalities from different abstraction levels within their implementations in runtime.

Based on this approach and aiming to test the implemented CB-AODV routing protocol in final application contexts, in this work an evaluation and diagnosis support tool for in-field deployment activities is proposed. The main target is to provide users with a set of functionalities to compare the behavior of the real deployment with what is expected from the simulation results. A general overview of the proposed architecture is shown in fig. 2, in which a combination of hardware and software components is integrated in a unique and novel in-field evaluation platform. The kernel of the tool is composed of a mobile device which contains the top level application and with which users can move in the target field in order to analyze specific deployment areas. The integration of a commercial AndroidOS-based Tablet connected to a Cookie-based platform that implements the corresponding WSN communication protocol is the basis of the aforementioned architecture. This configuration aims not only to experiment with IEEE 802.15.4 and protocols on top of it, but also to take advantage of the modularity of the Cookie platform by replacing the communication module with other stacks, such as ZigBee or 6LowPAN. The second important part of the implementation is the inclusion of a diagnosis component into the wireless nodes in order to gather information of the deployment from the evaluation tool. This component is able to extract information of the HW-SW implementation from different abstraction levels and forward it to the evaluation tool. Hence, real data can be analyzed in runtime in order to compare the performance of the simulated models with the behavior of the network.

Therefore, the proposed evaluation tool covers two mayor issues: routing protocol analysis of the implemented CBAODV as well as network connectivity and performance evaluation of an in-field WSN deployment under real conditions. The idea is not only to verify the effectiveness of the routing protocol, but also to study those factors that can affect the performance of the network connectivity and node's coverage, which can thus help users to integrate or customize different metrics depending on the type of network to be finally deployed.

\subsection{System implementation - considerations and features}

As shown in fig. 2, the integration between both platforms is made by means of using a serial-interface-based connection, through the available USB of the mobile device. This configuration schema aims to help users during the deployment and validation stages of the network, by providing them with a mobile device that supports WSN communication protocols (especially targeting IEEE 802.15 .4 based networks) and guides throughout the actions to be performed in-situ.

On the other hand, in order to carry out the node evaluation tasks in runtime, a set of software support libraries for network diagnosis is proposed, which is to be included in the HW-SW platform of the Cookie nodes. These components have been created in order to gather relevant data from the deployed nodes by accessing different parameters and configurations of the node implementation in an independent fashion, which means that the evaluation tool can extract information of specific node functionalities while the WSN application is running, thus without interfering in the node behavior. In order to do that, the proposed components are implemented on top of the PHY-MAC layers of the IEEE 802.15.4, so that the evaluation tool does not affect the behavior of the routing protocol since the communication from the tool to the nodes is done from a lower abstraction level. This implies that the main requirement to be addressed is the capability of being within the radio coverage of the target or, in case of carrying out a diagnosis task that includes more than one node, having coverage with at least one node of the target group. Hence, the main idea is to be as less intrusive as possible during the performance evaluation. This approach gives another important feature to the evaluation tool usage, which is to act as an in-situ "sniffer" device or packet analyzer, by gathering protocol frames regarding exchanged information among the nodes. Therefore, a MAC frame decoder is also included in the proposed functionalities, containing a format configuration as well as a network packet classification. The main parameters that are considered for the in-situ performance evaluation of the routing protocols and network connectivity are the followings:

Quality of the interconnections and coverage of nodes. A complete network connectivity schema is generated based on an iterative process in which every node gathers bidirectional information related to their corresponding neighbors/pairpoints in terms of quality of the link and radio signal strength. A routing map is also generated based on the path discovery between remote pair-points. This information can be compared and correlated with simulation models in order to detect possible mismatches between actual and modeled

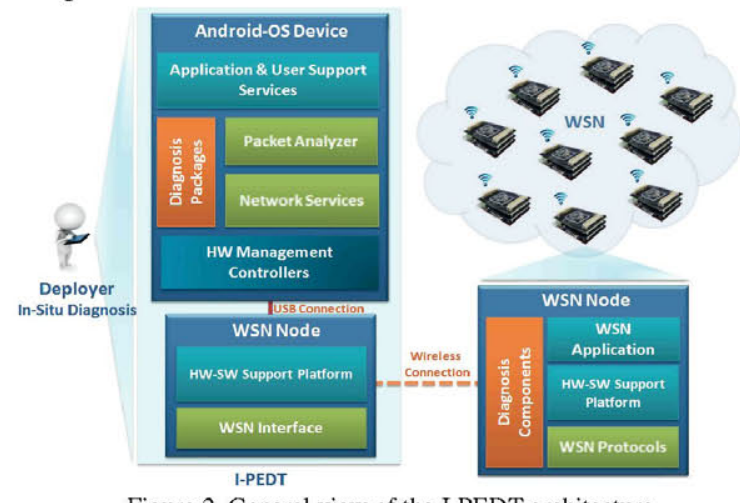

Figure 2. General view of the I-PEDT architecture. 
links/paths. Moreover, the routing map can be compared with the connectivity/MAC level in order to evaluate the performance of the routing protocol in terms of efficiency on the metric usage, asymmetry on the remote-pair-points, node isolation, congestion, and loops avoidance.

Metrics. As previously explained in the routing protocol implementation, several metrics have been included for the discovery process. The performance of these metrics in specific application scenarios can be gathered and evaluated based on the comparison of the runtime routing/network map generation and their theoretical calculation.

Sent/received packet ratio, packet loss rate (PLR). A packet delivery test is proposed to evaluate the performance of the routing capabilities as well as parameters regarding path discovery/rediscovery and route latency. The evaluation tool can launch this test by configuring the remote pair-points to be assess (having coverage with at least one of them) and the number of packets to be transmitted. The diagnosis components of the related nodes calculate the PLR and the result is then presented and stored in the corresponding database of I-PEDT.

Route Rediscovery Rate $(R D R)$ - link failure. Every node computes the number of rediscoveries by storing the information of the next hop with which a failure comes out (Note that each node only stores coverage information of their neighbor list). This process can help users to detect possible link problems in specific deployment areas.

Power consumption. The power consumption is measured in order to monitor the behavior of the nodes in terms of energy usage, so that battery levels and the consumed current can be analyzed to establish their impact and relationship with the communication performance of the nodes.

PHY-MAC layer assessment. By using the sniffer/packet analyzer capabilities, low level MAC frames can be monitored in order to evaluate the generation/reception of the packets from a lower abstraction level, so that problems regarding medium access and frame configuration can be detected.

Sensor measurements, RF configuration, node configuration. Key parameters regarding communication and internal peripheral configurations can also be obtained by using the evaluation tool, in order to study the correlation of particular set-ups with the behavior of the deployed devices.

The relative position of the device in terms of radio coverage is generated based on an in-field node scanning, so that the area of interest is evaluated in order to detect the surrounding nodes upon which I-PEDT can act on. Specific diagnosis frames have been defined along with their corresponding configuration fields and task IDs, based on the aforementioned parameters. The user then selects the corresponding node/group-of-nodes to act on, and the evaluation performance task is automatically generated and encoded to be wirelessly sent (802.15.4) to the target device.

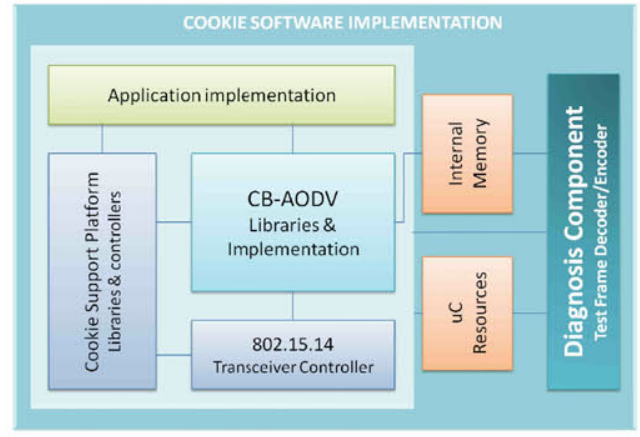

Figure 3. CB-AODV System Implementation.

\section{EXPERIMENTAL RESULTS \& USE CASES}

The proposed CB-AODV routing protocol has been tested and analyzed in a real WSN indoor deployment at the Center of Industrial Electronic by means of using the capabilities of the I-PEDT, in order to validate the designed functionalities and components under the Cookie HW-SW architecture. The implementation of the CB-AODV routing protocol has been carried out based on the Cookie support platform proposed in [16], integrating the software components as shown in fig. 3. In terms of memory usage, CB-AODV was included in the Cookie processing layer that incorporates an ADuC841 microcontroller from Analog Devices [17], which provides up to $64 \mathrm{~KB}$ of flash/program memory as well as $2 \mathrm{~KB}$ of data storage $+4 \mathrm{~KB}$ of Flash/EE memory (the later one reserved for creating and keeping the routing tables in runtime). The final implementation fits in $8 \mathrm{~KB}$ of program memory and less than $2 \mathrm{~KB}$ of data memory (including the support software components for the diagnosis capabilities), which gives enough free memory space for Cookie-based application proposals. From the point of view of the Performance Evaluation Tool I-PEDT, the implementation has been carried out by using an Asus Transformer Tablet that includes the Android Ice Cream operating system connected to a Cookie node through the USB socket, so that all the diagnosis capabilities and GUIs were designed using the Java-based coding features of such OS, as shown in fig. 4, in which the main features of the tool interface are highlighted. This configuration schema also provides the possibility to easily port the implementation to other Android-based devices.

Two of the topologies used to test the CB-AODV are shown in fig. 5. The experiments were set up based on three main configurations executed by I-PEDT. First of all, in order to verify the expected behavior of the routing protocol and its robustness throughout a period of time, nodes were configured to send packets every second to a random address. The packet loss rate and the network/routing map were then computed by the evaluation tool in order to obtain the degree of success during the transmission/reception process as well as the routing discovery procedure in such conditions. The TX power of the radio transceivers was also configured to modify the node coverage, so that the routing capabilities are evaluated by creating paths with the maximum amount of hops as possible, which helps developers to assess the performance of the integrated metrics. In this way, as a second test case, one of the nodes was configured by the I-PEDT to send 100 packets with a frequency of one message per second (these parameters can be reconfigured by the tool) to its farthest node, i.e., a data exchange from the two most distant points of the network (node 1 and 9 in the figure). The main goal was to analyze the performance of both the PLR and the included metrics, specially the PATH-DR and the ETX, which depend on the

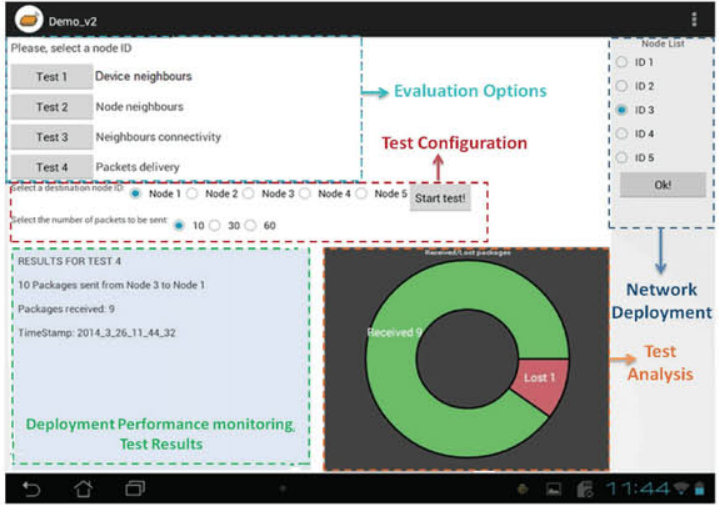

Figure 4. General view of the I-PEDT User Interface. 

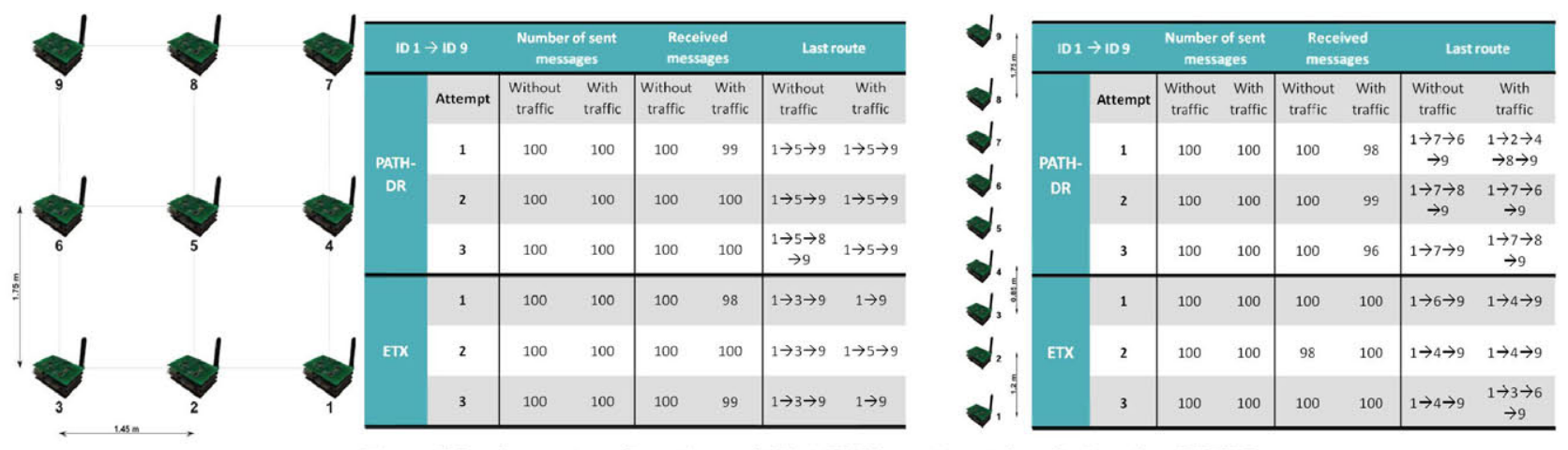

Figure 5. Deployment configuration and CB-AODV experimental results based on I-PEDT.

link quality parameter. Furthermore, a third test case was also carried out in this scenario, in which a "crowded environment" was set up by configuring the rest of the nodes to send packets to random addresses, while the two distant nodes continued exchanging data between each other. Metrics and the test configurations were launched by using the evaluation tool, and an iterative execution was done three times per configuration in order to obtain the information of the network behavior.

In fig. 5, the main results regarding the proposed scenarios are classified according to the applied metric and the differences in traffic conditions, in addition to the routing paths obtained by means of using the tool. The percentage of received packets is very close to $100 \%$, being slightly inferior in the case of traffic conditions. As a result of comparing the metric performances, the ETX has fewer hops than the PATHDR, as expected (because ETX penalizes the number of hops). However, the percentage of messages that the final node receives is not higher in case of the PATH-DR than with the ETX, as it would be expected. Although both metrics have similar results, it is important to consider a greater amount of nodes to analyze their impact within extended application scenarios, with more hops and alternative points per path.

Another experimental use case has been performed in order to verify the usability of I-PEDT in heterogeneous WSNs in terms of hardware and software platforms, by evaluating the implemented packet analysis capabilities of the tool. In this way, a WSN deployment composed of two different platforms was carried out. The first one is a custom TelosB-based node which includes TinyOS as the running software platform, and the second one is the Cookie node with its corresponding custom software support platform. Sensor nodes periodically send packets to a coordinator node and I-PEDT allows deployers to evaluate in-situ whether the communication between the heterogeneous platforms is properly done by gathering and analyzing the low level packet frames regarding data, control and acknowledge messages, as shown in fig. 6 .

\section{CONCLUSIONS \& CONTRIBUTIONS}

In this work, two major approaches for the efficiency of WSN deployments under real applications have been proposed focused on studying, analyzing and evaluating the performance of routing protocols in realistic environments. A modified AODV-based routing protocol (CB-AODV) for a

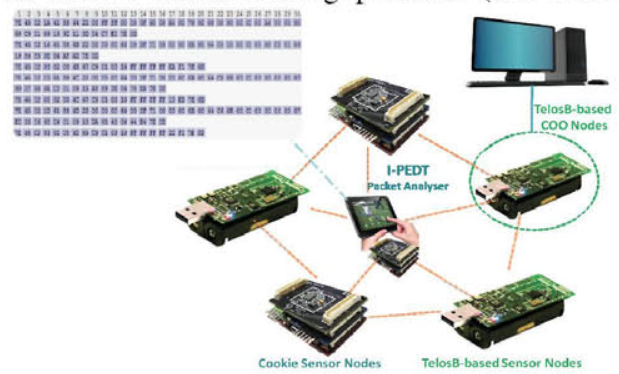

Figure 6. Network traffic analysis in heterogeneous deployment. reactive and flexible Ad-hoc network configuration has been fully implemented and tested into a modular HW-SW platform, which copes with the limitation of resource constraints and memory budget during the design and implementation stages. A complete In-situ Performance Evaluation and Diagnosis Tool (I-PEDT) was also proposed as a major contribution not only for the CB-AODV assessment, but also to provide users with an in-field network analysis tool for optimizing and validating node functionalities under real application conditions. In order to put both CB-AODV and IPEDT into various application contexts, the next step is to expand their applicability to diverse use cases where the amount of nodes to be deployed can be greater and scalable, so that deeper analysis on link failures, latency problems and metric efficiency will be studied in an extended environment.

\section{REFERENCES}

[1] D. Goyal, M. Tripathy, "Routing protocols in wireless sensor networks: A survey," in proc. ACCT'12, pp. 474-480, Jan. 2012.

[2] K.L. Pang, Y. Qin, "The comparison study of flat routing and hierarchical routing in ad hoc wireless networks, " in Proc. ICON'06, vol. 1, pp. 1-6, Sept. 2006.

[3] N. A. Pantazis, S. A. Nikolidakis, D. D. Vergados, "Energy-Efficient Routing Protocols in Wireless Sensor Networks: A Survey," IEEE Communications Surveys Tutorials, vol. 15, no. 2, pp. 551-591, 2013.

[4] M. Chen, V. Leung, S. Mao, Y. Xiao, I. Chlamtac, "Hybrid Geographical Routing for Flexible Energy-Delay Trade-Offs," IEEE Trans. Veh. Technol., 2009, Vol. 58, Issue 9, pp. 4976-4988.

[5] S. Mohseni, R. Hassan, A. Patel, and R. Razali, "Comparative review study of reactive and proactive routing protocols in MANETs," in proc. DEST'10, April 2010, pp. 304 -309.

[6] J. Portilla, A. de Castro, E. de la Torre, T. Riesgo, "A Modular Architecture for Nodes in Wireless Sensor Networks" in JUCS, vol. 12, n 3, pp. 328-339, March 2006.

[7] A. Sobeih, J.C. Hou, L.-C. Kung, N. Li, H. Zhang, et al. "J-Sim: A Simulation and Emulation Environment for Wireless Sensor Networks," IEEE Wireless Comm., vol. 13, no. 4, pp. 104-119, Aug. 2006.

[8] A. Karatzoglou, M. Scholz, T. Riedel, M. Beigl, "A prototype of an insitu radio sensing and visualization device," in Proc. INSS 2012.

[9] L. Selavo, G. Zhou and J. A. Stankovic "SeeMote: In situ visualization and logging device for wireless sensor networks," in Proc. BROADNETS'06, pp.1 -9, 2006.

[10] IEEE Standard Part 15.4: Wireless Medium Access Control (MAC) and Physical Layer (PHY) Specifications for LR-WPANs, 2003.

[11] CC2420 2.4 GHz. Chipcon products from Texas Instruments.

[12] C. Perkins, E. Belding-Royer and S. Das, Ad hoc On-Demand Distance Vector (AODV) Routing. RFC 3561, 2003.

[13] C. Gomez, A. Boix, J. Paradells, "Impact of LQI-based routing metrics on the performance of a one-to-one routing protocol for IEEE 802.15.4 multihop networks". EURASIP J. Wireless Commun. Netw. 2010, 1-20.

[14] C. Gomez, P. Salvatella, O. Alonso, J. Paradells, "Adapting AODV for IEEE 802.15.4 Mesh Sensor Networks: Theoretical Discussion and Performance Evaluation in a Real Environment," in Proc. WoWMoM'06, Niagara-Falls, Buffalo-NY, 26-29 June 2006.

[15] R. J. v. Glabbeek, P. Höfner, W. L. Tan, M. Portmann, "Sequence numbers do not guarantee loop freedom: AODV can yield routing loops," in Proc. MSWiM'13, NY, USA, Nov. 2013, pp 91-100.

[16] G. Mujica, V. Rosello, J. Portilla, T. Riesgo, "Hardware-software integration platform for a WSN testbed based on cookies nodes", in proc. IECON 2012. pp. 6013-6018.

[17] Analog Devices, http://www.analog.com/en/processors-dsp/analogmicrocontrollers/aduc841/products/product.html. 